\section{Presumed paraneoplastic brainstem encephalitis secondary to ovarian teratoma}

Sir,

Ovarian teratomas are rarely associated with paraneoplastic central nervous system (CNS) disorders, especially in adolescent girls. ${ }^{[1,2]}$ Benign ovarian teratomas have been associated with paraneoplastic CNS disorders in only two cases, one each of paraneoplastic brainstem encephalitis (PNBE) and paraneoplastic encephalomyelitis. ${ }^{[3,4]}$

We present the second case of PNBE associated with a benign ovarian teratoma and the first in an Indian adolescent.

A 15-year-old girl was evaluated for intractable vomiting of four weeks duration. Examination revealed an abdominal mass and ultrasound of the abdomen suggested a juxta-ovarian mass. She underwent laparoscopic surgery and complete removal of the mass. Histopathology revealed a mature ovarian teratoma with cystic components. Her vomiting persisted one week after surgery. Postoperative abdominal ultrasound and upper gastrointestinal (GI) endoscopy were normal. Two weeks after surgery, she complained of difficulty in walking and slurred speech. On examination, she had dysarthria and a wide-based gait. Routine blood tests were normal. Non-contrast magnetic resonance imaging (MRI) brain and nerve conduction studies were normal. A repeat contrast MRI two weeks later was also normal. Cerebrospinal fluid examination was normal. At this point, paraneoplastic brainstem encephalitis was considered. She was given a course of IV methylprednisolone (IVMP) $1 \mathrm{~g} /$ day for five days. Her vomiting abated after three days. Her ataxia and dysarthria improved slowly and she was able to ambulate. Four weeks after treatment, she was able to function normally with minimal residual dysarthria. Her gait ataxia had nearly disappeared. The parents were unwilling for paraneoplastic antibody screening (anti Ro and anti La). At follow up, one and a half years later, she was asymptomatic.

Our case is unique in two respects. It is the first case of presumed paraneoplastic neurological disorder associated with an ovarian teratoma reported from India. Secondly the presenting manifestation of paraneoplasia was intractable vomiting. Only after other cerebellar signs developed was it possible to diagnose a paraneoplastic syndrome.

Before diagnosing a paraneoplastic disorder, it is mandatory to rule out other conditions such as CNS metastases, infections, chemotherapy or radiation-induced symptoms, loco-regional tumor complications, autoimmune conditions and CNS demyelination. An MRI study helps in excluding many of these conditions.

Detection of antibodies that co-localize with EFA6A may be helpful in the diagnosis of these syndromes. ${ }^{[5]}$ However, in a developing economy like ours, these tests are expensive and the cost saving can often be utilized for therapy. This case report emphasizes the necessity of considering a PNBE when neurological symptoms or intractable vomiting develop in the presence of an ovarian teratoma.

It should be noted that steroid responsiveness was observed in an earlier case of paraneoplastic encephalomyelitis associated with a benign ovarian teratoma. ${ }^{[4]}$

Steroid responsivity and prolonged or permanent remission seem to be a feature of PNBE associated with ovarian teratomas. Clinicians should consider steroids when teratoma removal alone does not result in alleviation of symptoms.

K. Parameswaran, Boby V. Maramattom ${ }^{1}$

Department of Neurology, Indo-American Hospital Brain and Spine center, Vaikom, 'Department of Neurology, Lourdes Hospital, Kochi, Kerala, India. E-mail: bobvarkeys@yahoo.com

\section{References}

1. Lee AC, Ou Y, Lee WK, Wong YC. Paraneoplastic limbic encephalitis masquerading as chronic behavioural disturbance in an adolescent girl. Acta Pediatr 2003;92:506-9.

2. Stein-Wexler R, Wootton-Gorges SL, Greco CM, Brunberg JA. Paraneoplastic limbic encephalitis in a teenage girl with an immature ovarian teratoma. Pediatr Radiol 2005;35:694-7.

3. Muni RH, Wennberg R, Mikulis DJ, Wong AM. Bilateral horizontal gaze palsy in presumed paraneoplastic brainstem encephalitis associated with a benign ovarian teratoma. J Neuroophthalmol 2004;24:114-8. 


\section{Letters to Editor}

4. Taylor RB, Mason W, Kong K, Wennberg R. Reversible paraneoplastic encephalomyelitis associated with a benign ovarian teratoma. Can J Neurol Sci 1999;26:317-20.

5. Vitaliani R, Mason W, Ances B, Zwerdling T, Jiang Z, Dalmau J.
Paraneoplastic encephalitis, psychiatric symptoms and hypoventilation in ovarian teratoma. Ann Neurol 2005;58:594-604.

Accepted on 25-09-2007 